\title{
Dynamical Observation of Heat Flow and the NDI in a Simulated Welded Zone using Active Thermography with a Coolant Material
}

by H. Endoh* and T. Hoshimiya**

\begin{abstract}
*Dept. of Mechanical Engineering, Tohoku Gakuin University, 13-1, Chuo 1, Tagajyo, 985-8537, Japan, enpal@tjcc.tohoku-gakuin.ac.jp

**Dept. of Electronic Engineering, Tohoku Gakuin University, 13-1, Chuo 1, Tagajyo, 985-8537, Japan, tpth@tjcc.tohoku-gakuin.ac.jp
\end{abstract}

\begin{abstract}
An active thermographic imaging system was constructed, in which a cooling material contacts the surface and scans over a welded zone of two stainless plates. Both a time-varying thermographic image and a real-time response of each pixel were recorded. A thermal image showed that the heat flow from the welded zone toward the cooling material apparently forms an isolated high-temperature region which indicates the existence of a subsurface simulated welding defect. This method is a simple, easy and high-precision nondestructive inspection (NDI) technique using active thermography.
\end{abstract}

\section{Introduction}

At present, spot welding is popularly used in applications such as assembly of automobiles or industrial products. However, in these products small invisible undersurface defects are expected to be contained. Activethermography or photothermal radiometry has become more and more important in nondestructive inspection (NDI) tool $[1,2,3]$ for these industrial products.

Due to the development of real-time thermography, NDI of these products with thermotracer using light or electrical source became important. In this study, the thermographic instrumentation system, in which a gelated coolant scans over rear-surface of the specimen, and the real-time response of the thermal image was recorded with a thermotracer located at the front surface of the specimen. The details of the present method were described to present a simple, easy and high-precision NDI tool using active thermography.

\section{Experimental apparatus and specimens}

Two types of scanning scheme were presented. In the former type, a coolant material moves along a direction of heat flow. In the latter case, it moves along a perpendicular direction to the heat flow from the undersurface defect to the surface of the specimen.

\subsection{Axial movement of the coolant material}

The experimental setup for the first one is shown in figure 1 (a). The coolant material was made of agar and ethylene-glycol with length and diameter of $80 \mathrm{~mm}$ and $20 \mathrm{~mm}$, respectively. It was stored in a refrigerator below 0 degree and used to contact the specimen. A thermal image was observed by a thermo-tracer (NEC San-ei, TH 9100) with a temperature resolution of $0.06 \mathrm{~K}$. The thermal image was recorded by a PC connected with an IEEE 1394 bus. The realtime (30 flames/sec) thermal response was recorded by a PC.

\subsection{Lateral movement of the coolant material}

The experimental setup for the second one is shown in figure 1 (b). The same scanning apparatus was used as the axial movement scheme. Linear slider carried a coolant material toward the specimen, contacted and kept contacting one second, and then returned to the original position.

\subsection{Specimens under study}

The specimens were made of welded two stainless steel (SUS316) plates. In order to simulate a welded specimen with an undersurface defect, rectangular hole in a stainless plate or spacing were made between two plates, as shown in figure 2. A hole with a diameter of $0.5 \mathrm{~mm}$ was drilled at the center of the $6 \mathrm{~mm} \times 6 \mathrm{~mm}$ square zone. A gelated coolant material (agar and ethylene glycol, about $-15^{\circ} \mathrm{C}$ )) contacted with the specimen (welded stainless steel plates) acted as a heat sink. Experiments were carried out at room temperature (about $25^{\circ} \mathrm{C}$ ). 


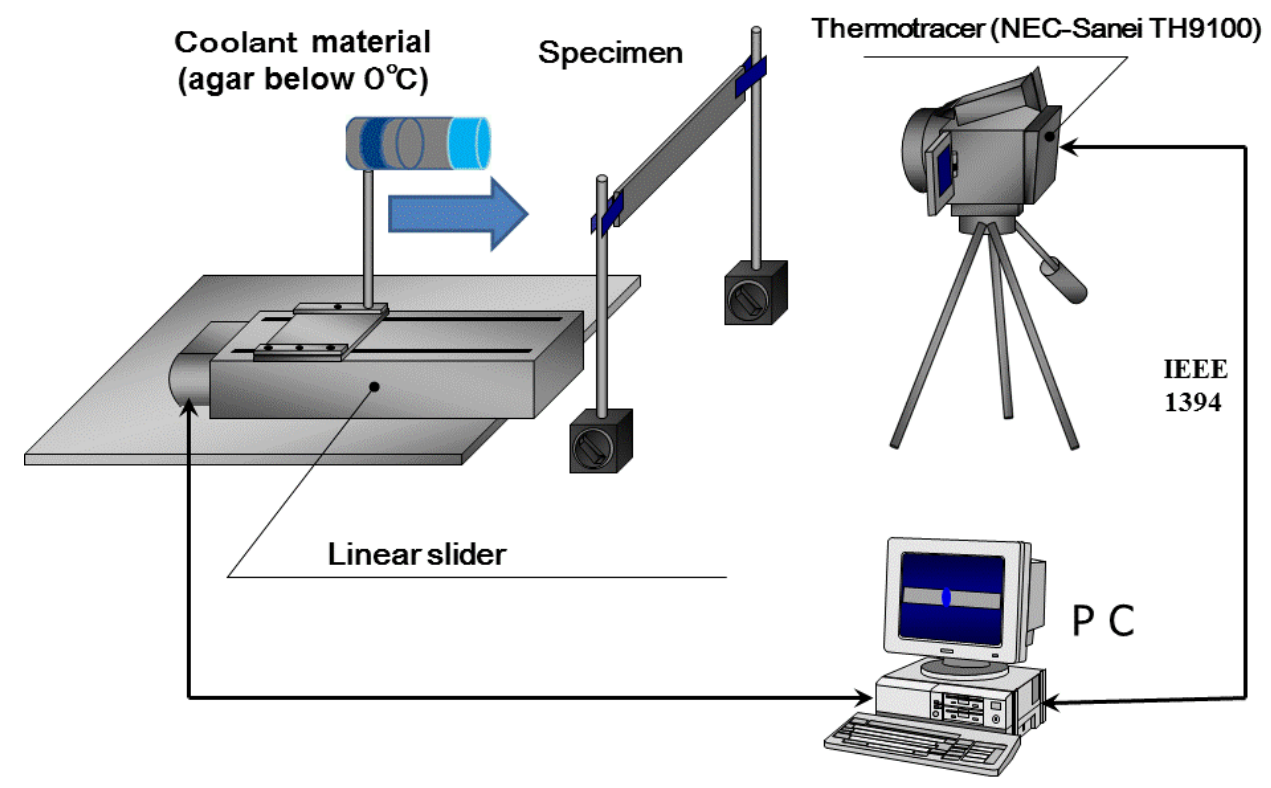

(a) Axial movement of a coolant material

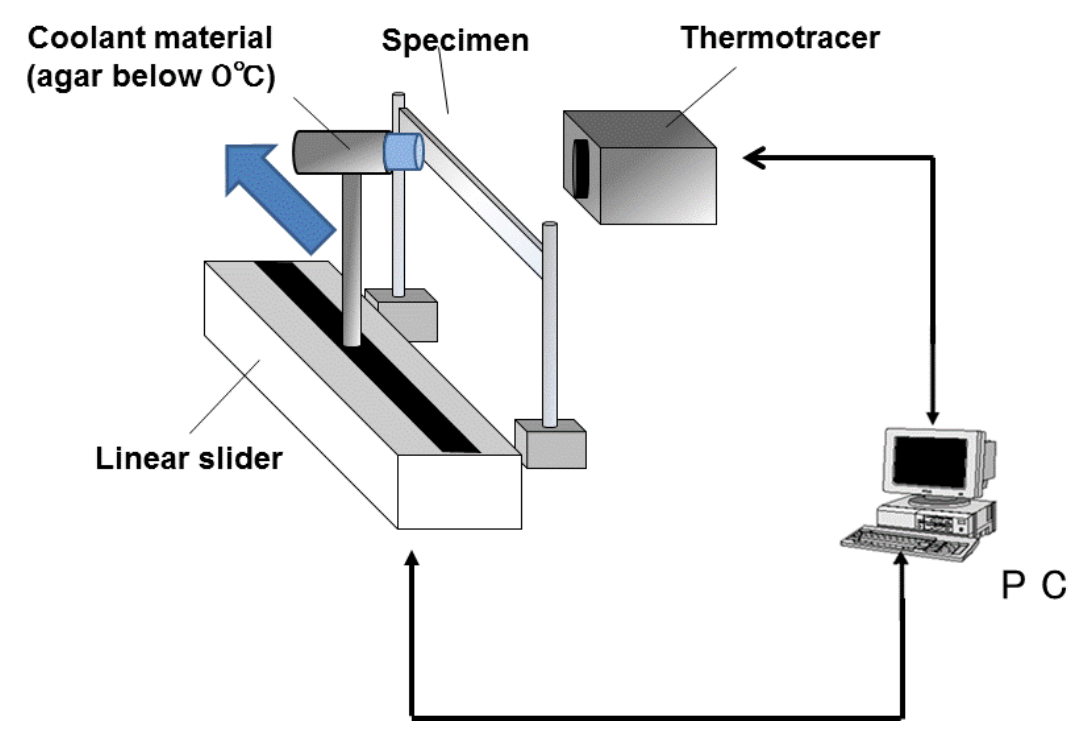

Fig. 1. Experimental setup (b) lateral movement of a coolant material 


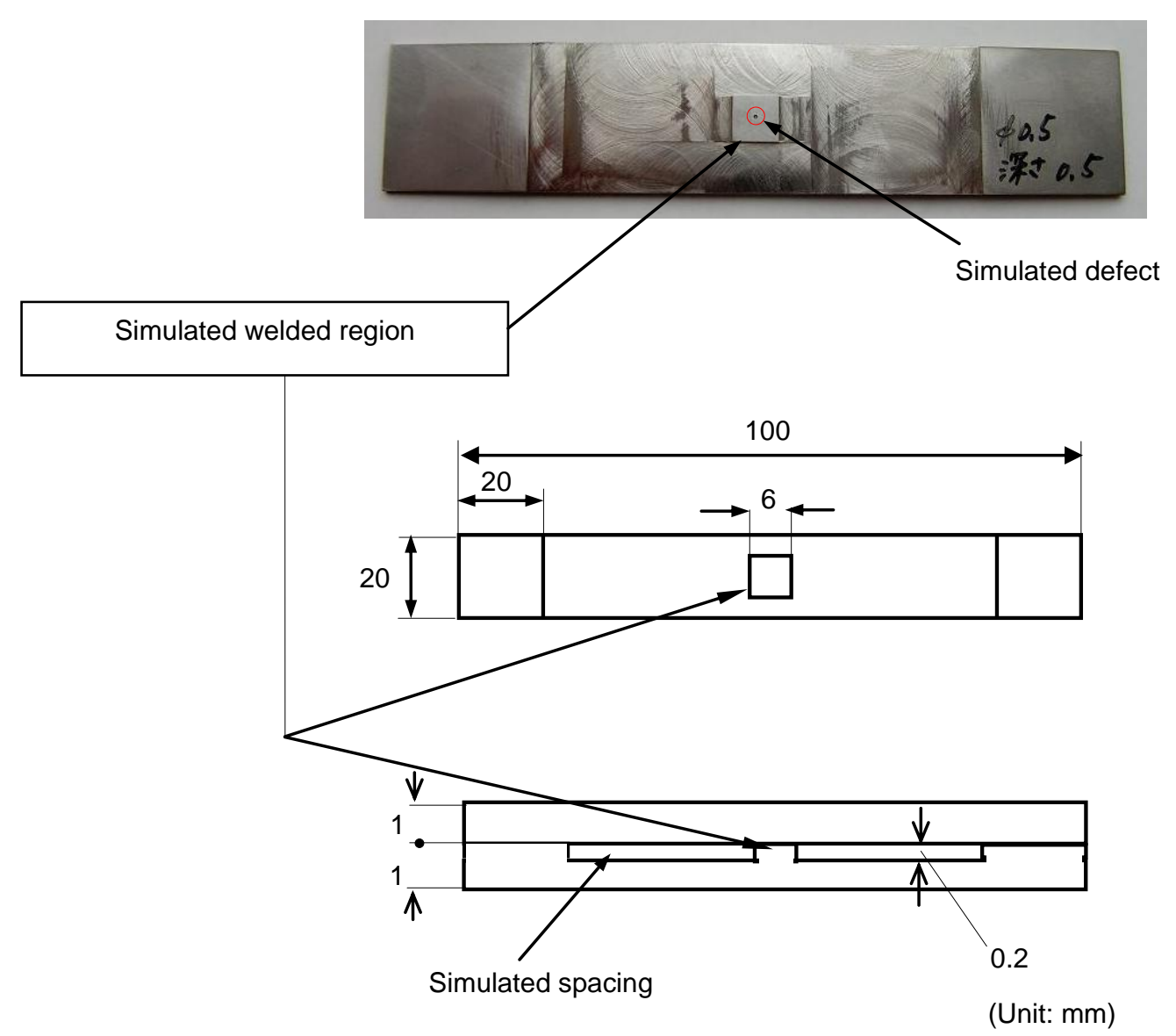

Fig. 2. Simulated welded specimen with an undersurface defect

\section{Experimental results}

The thermal image obtained at the moving speed of $10 \mathrm{~mm} / \mathrm{s}$ was shown in figure $3(\mathrm{a})$. On the other hand, temperature profile along A-A' and B-B' lines were shown in figures 3 (b) and (c), respectively. At the center of the profiles high-temperature region was apparently observed, which corresponds to the undersurface defect in the simulated welded region. The thermal image obtained at the moving speed of $50 \mathrm{~mm} / \mathrm{s}$ was shown in figure 4 (a). Figure 4 (b) shows temperature profile along $A-A^{\prime}$ line of the thermal image in figure 4 (a). In figure 4 (b), it is found that the locally high-temperature spike in low-temperature region of the simulated welding region was observed as well as figures 3 (b) and (c). Scanning speeds ranging from $10 \mathrm{~mm} / \mathrm{s}$ to $100 \mathrm{~mm} / \mathrm{s}$ with the increment of $10 \mathrm{~mm} / \mathrm{s}$ were performed, and in the cases of $10-50 \mathrm{~mm} / \mathrm{s}$ the high-temperature spikes (circled area in figures 3 (b), (c) \& 4 (b)) in thermal image profile indicating the existence of the undersurface defect were observed clearly. 


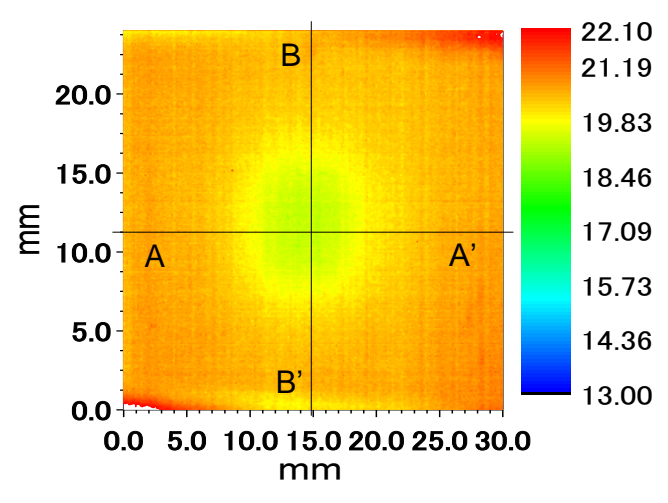

(a)

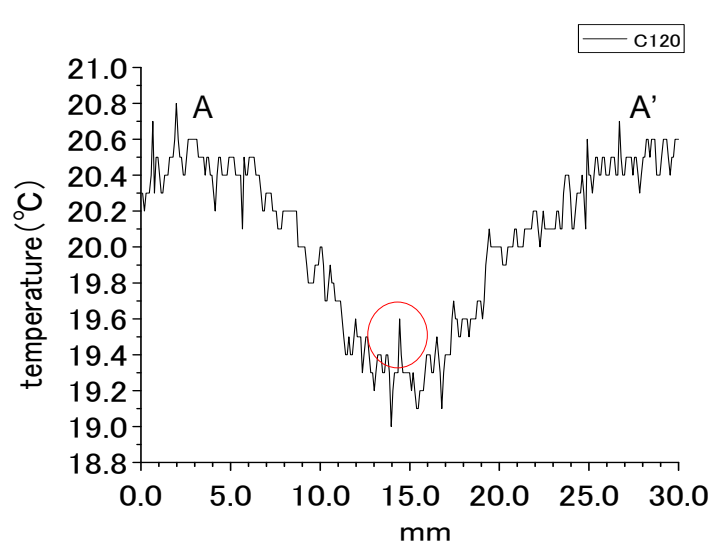

(b)

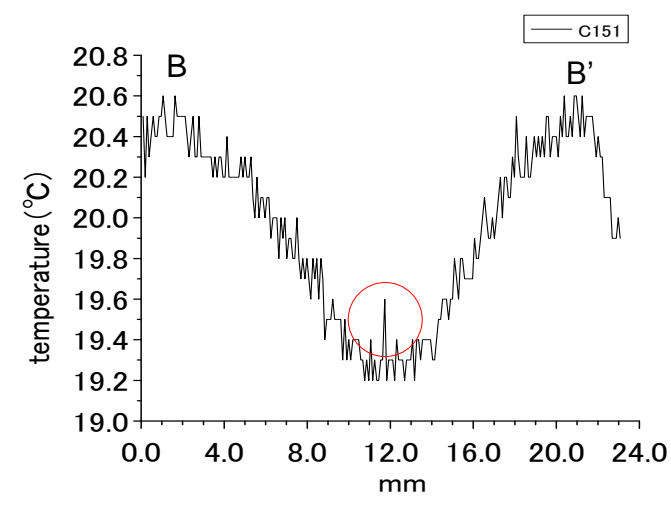

(c)

Fig. 3. (a)Thermal image, (b) temperature profile along $A-A$ ' line, (c) temperature profile along $B-B$ ' line $(v=10 \mathrm{~mm} / \mathrm{s})$. 


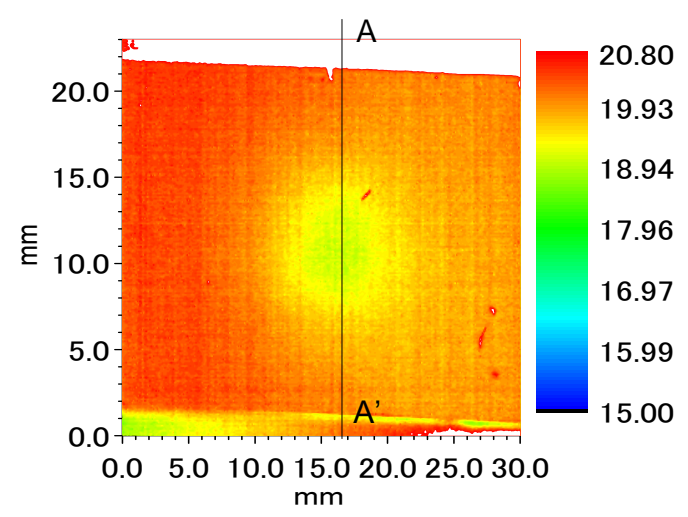

(a)

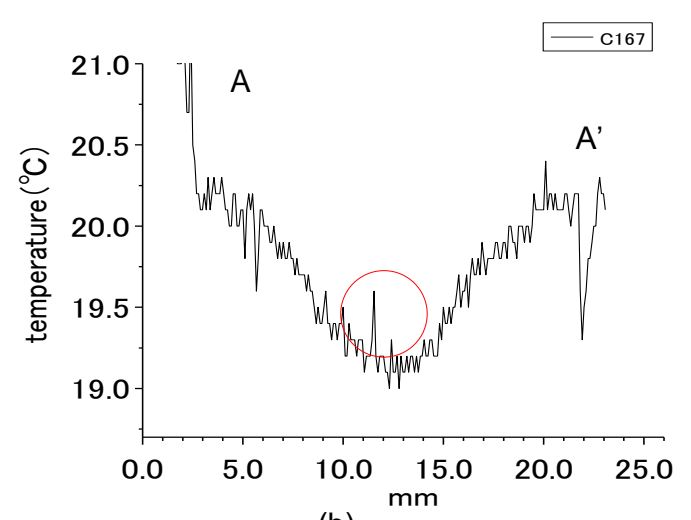

(b)

Fig. 4. (a)Thermal image, (b) temperature profile along $A-A$ ' line $(v=50 \mathrm{~mm} / \mathrm{s})$.

\section{Discussion and conclusion}

Documents In this section, qualitative discussion on the thermal images obtained with axial and lateral movements. Since the heat-diffusion equation is invariant under the transformation:

$$
\mathrm{T} \rightarrow-\mathrm{T} \text { and } \vec{r} \rightarrow-\vec{r}
$$

(heating is changed to cooling with space-reversal, where $\mathrm{T}$ means the temperature deviation from the ambient temperature), the temperature distribution in cooling of material under an axially-symmetric is similar to that in heating of the same material.

\subsection{Axial movement temperature profile}

In the case of axial movement of coolant material, axial symmetry of heat transfer was conserved so that the heat inside and the surface of the specimen transfers toward the interface surface of the specimen to the coolant. The artificially-made inner defect inside the specimen reflects the heat flow so that the reflected thermal wave results a spikeshaped high-temperature region as shown in the figure.

\subsection{Lateral movement temperature profile}

In the case of lateral movement of coolant, axial symmetry was broken so that the temperature distribution at the surface $z=0$ is described as $T(x, y, 0, t)$ rather than that of axial symmetry $T(r, z=0, t)$, where the moving direction is $x$. The diffusion of the heat along the direction $y$ (perpendicular to the moving direction $x$ ) is free from the coolant movement so that its spatial distribution is the same as that of axial movement. The spatial distribution of the temperature along the $x$ axis (the direction of coolant movement), on the other hand, is squeezed. As a result, temperature distribution $T(x, y, 0$, t) shows an elliptical shape as shown in Figure 3 (a).

In the case of scanning speeds ranging from $60 \mathrm{~mm} / \mathrm{s}$ to $100 \mathrm{~mm} / \mathrm{s}$, the high-temperature region spike corresponding to the undersurface defect have obtained with embedded in thermal noise. To improve the $\mathrm{SN}$ ration, a synchronous recording technique with a starting time of the linear slider is favorable. In the present paper, only the 
thermal image at the particular time was shown. However, the present system enables to utilize the time-domain observation. An active thermographic imaging system with external I/O control with a control software "ir-motion" was in progress, and the averaging of the thermal images will improve the SN ratio.

In conclusion, a thermographic measuring system with both axial and lateral movement of the coolant material was designed and fabricated with a time-varying thermal recording in this study. The present system was easily applicable to the NDI of spot welding used whole over the world.

\section{REFERENCES}

[1] P.V.McLaughlin,Jr, M.G.Mirchandani, P.V.Ciekurs, "Infrared Thermographic Flaw Detection in Composite Laminates", Journal of Engineering Materials and Technology, vol. 109, Apr., pp. 146-150, 1987.

[2] Robert A. Buchanan, Paul Condon, Lee Klynn, "Recent Advances in Digital Thermography for Nondestructive Evaluation", SPI, vol. 1313, Thermosense XII, 1990, pp. 134-142, 1990.

[3] C., Yu-Lin Wang, Fu-Zhong Shen, Shan-Tung Tu, D, "A Study of Creep Crack Propagation of HK 40 Furnace Tubes with C-Shaped Specimens", Engineering Fracture Mechanics, vol. 47 no. 1, pp. 39-47, 1994. 